\title{
The effects of dietary lipids on dendritic cells in perinodal adipose tissue during chronic mild inflammation
}

\author{
Christine A. Mattacks, Dawn Sadler and Caroline M. Pond ${ }^{*}$ \\ Department of Biological Sciences, The Open University, Milton Keynes MK7 6AA, UK
}

(Received 10 November 2003 - Revised 16 February 2004 - Accepted 18 February 2004)

\begin{abstract}
The effects of dietary lipids on the abundance of dendritic cells in adipose tissue in anatomically defined relationships to chronically inflamed lymph nodes were investigated in mature male rats fed plain chow or chow plus $20 \%$ sunflower-seed or fish oil. The popliteal lymph nodes were stimulated by local subcutaneous injection of $20 \mu \mathrm{g}$ lipopolysaccharide to both hindlegs three times/week for 2 weeks. The masses of the major adipose depots and the numbers of dendritic cells emerging from perinodal adipose tissue and samples 5 and $10 \mathrm{~mm}$ from the popliteal lymph nodes were measured, and those from omental and mesenteric adipose tissue around and remote from lymphoid tissue, and mesenteric and popliteal lymph nodes. Dendritic cells were most numerous in the perinodal adipose tissue, with the corresponding 'remote' samples containing 25-50\% fewer such cells under all conditions studied. Dietary sunflower-seed oil increased the numbers of dendritic cells by about $17 \%$ in all adipose samples and fish oil reduced the numbers in perinodal tissue by about $5 \%$. The fish-oil diet diminished responses of the intra-abdominal adipose depots to local stimulation of the popliteal node. Correlations in dendritic cell numbers were stronger between perinodal samples from different depots than between remote and perinodal samples from the same depot and after the sunflower-seed-oil diet compared with fish oil. These data show that dietary lipids modulate the number of dendritic cells in lymphoid tissue-containing adipose depots and support the hypothesis that perinodal adipose tissue interacts locally with lymphoid cells.
\end{abstract}

Popliteal: Mesenteric: Omental: Fish oil: Sunflower-seed oil: Lymph nodes

Dendritic cells (DC) are immune cells that permeate many non-neural tissues, migrating to and from lymph nodes and other lymphoid structures (Huang et al. 2000). Their activities and properties are regulated by various cytokines and by fatty acid-derived messenger molecules (Schreiner et al. 1988; Luft et al. 2002; Morelli \& Thomson, 2003). Prostaglandins and leukotrienes are mostly short-lived molecules acting in an autocrine or paracrine mode. The best known are derived from arachidonic acid (20:4n-6) (Bagga et al. 2003), but some, and the more recently described resolvins, are metabolites of the $n-3$ family of long-chain PUFA (Serhan et al. 2002). The immediate source of both classes of fatty acid precursors is membrane phospholipids, but these essential fatty acids derive ultimately from the diet. DC membranes are extensive and highly dynamic, being remodelled by movements and by endocytosis (Levine \& Chain, 1992). The DC responses to microbial lipoproteins are mediated by toll-like receptors (Hertz et al. 2001), that are themselves modulated by dietary PUFA (Lee et al. 2003). Like many other kinds of immune cells, DC readily incorporate dietary fatty acids (Calder, 2001), though little is known about exactly where and how the transfer takes place.

Mammalian lymph nodes are surrounded by perinodal adipose tissue that has many special properties that enable it to interact locally with adjacent lymphoid tissues (Pond, 2003a,b). Immune cells permeate adipose tissue adjacent to lymphoid structures, especially during and after immune stimulation. Following the immunisation of mice experimentally infected with Helicobacter pylori, both $\mathrm{T}$ and $\mathrm{B}$ lymphocytes appear in the adipose tissue surrounding the stomach, though none can be found in the homologous site of uninfected or unimmunised mice (Mueller et al. 2003). During immune responses, newly formed cells in the stimulated lymph nodes selectively incorporate fatty acids derived from triacylglycerols in the surrounding adipose tissue (Pond \& Mattacks, 2003). As well as being modulated by diet, the composition of fatty acids in adipose tissue triacylglycerols depends upon the anatomical relationships of the samples to lymph nodes; the relative abundance of PUFA is highest in triacylglycerols in perinodal adipocytes and decreases 
with distance from the lymph nodes (Mattacks \& Pond, 1997).

DC secrete a wide range of cytokines, some of which are known to stimulate lipolysis in adipocytes adjacent to lymphoid tissues (Mattacks \& Pond, 1999; Pond \& Mattacks, 2002). DC infiltrating the adipose tissue may be among the lymphoid cells that can stimulate lipolysis in adjacent adipocytes (Pond \& Mattacks, 1995, 1998), and take up the fatty acids so released (Pond \& Mattacks, 2003), but the combination of fatty acids available to them depends upon which adipocytes they interact with. Some fatty acids serve as fuel, but others are incorporated into the DC, particularly their abundant membranes. DC probably die inside lymph nodes and the debris is taken up by other lymphoid cells (Hart, 1997), so these itinerant cells may mediate the transfer of fatty acids from perinodal adipocytes to proliferating lymphocytes (Pond \& Mattacks, 2003). To assess their role in this process, and to understand how DC acquire fatty acids of dietary origin, it is essential to know more about where these cells are in contact with adipocytes that are specialised to interact with them. In the present paper, we quantify DC in adipose tissue from sites defined by their anatomical relationships to lymph nodes and omental milky spots. We compare cell numbers in homologous tissues of rats fed diets enriched with fish oil (rich in $n$-3 PUFA), which is usually anti-inflammatory (Calder, 2001), or sunflower-seed oil (rich in $n-6$ PUFA) which has contrasting actions on immune processes.

The tissues chosen for study are popliteal and mesenteric lymph nodes and their associated adipose depots, and the omentum. The popliteal lymph nodes are quiescent in healthy animals but can be stimulated locally by minimally invasive procedures (Smith \& Morris, 1970). The pair of small adipose depots enclosing the popliteal nodes is very consistent in form, thus facilitating the identification of exactly homologous samples of fresh tissue, and is composed of adipocytes that interact strongly with lymph node lymphoid cells (Pond \& Mattacks, 1995). Mesenteric lymph nodes are large and quite numerous, and surrounded by perinodal adipose tissue that shows strongly the site-specific properties characteristic of interactions with lymphoid tissues (Pond \& Mattacks, 1995; Mattacks \& Pond, 1997, 1999; Mattacks et al. 2002). The omentum consists of adipose tissue interspersed with numerous milky spots that have some properties in common with small, simple lymph nodes (van Vugt et al. 1996). Various kinds of leucocytes migrate from milky spots to the peritoneum, and are activated by bacterial extracts (Cui et al. 2002).

Rats were chosen in preference to guinea-pigs or rabbits because murine DC markers are available commercially and may react efficiently only with species of the family Muridae. However, the mass of the popliteal adipose depots in adult rats is only about one-quarter of that of ad libitum-fed mature guinea-pigs (Mattacks et al. 2003), so to maximise the yield of tissue without resorting to procedures that induce obesity, only fully grown male rats were used. Another advantage of using such animals is that, in contrast to young, growing rats, they fatten very little when fed diets supplemented with edible oils, thus avoiding confusion between the effects of dietary lipids per se and the consequences of hypertrophy of adipose tissue.

\section{Materials and methods}

\section{Animals and tissue samples}

Male Sprague-Dawley rats ( $n$ 168), fed ad libitum on RM3 chow (Special Diet Services, Witham, Essex, UK), were aged about 6 months (i.e. fully grown) when the present experiment started. The total body mass of each rat was recorded at the start of the experiment and at killing. Three groups were fed for 6 weeks on unmodified RM3 chow (lipid content of $4.25 \%$ ) or chow supplemented with $20 \%$ sunflower-seed oil or $20 \%$ fish oil (raising the lipid content to $24.25 \%$ ). In one half of the rats of each dietary group, chronic immune disturbance was stimulated by injecting $20 \mu \mathrm{g}$ lipopolysaccharide (LPS) (L2630 from E. coli; Sigma, Poole, Dorset, UK) dissolved in buffered sterile saline, $\mathrm{pH} 7.4$ (injected volume about $0.1 \mathrm{ml}$ ) into both the lower legs (the 'catchment' area of the popliteal lymph nodes), three times weekly for 2 weeks including the day before killing. This immune-stimulation procedure produced no detectable signs of distress in the animals. The other half of each dietary group was designated 'unstimulated'.

After killing by $\mathrm{CO}_{2}$ anaesthesia, each rat was weighed and the epididymal, perirenal and retroperitoneal, mesenteric, omental, inguinal and popliteal adipose depots were dissected out at once, washed in Hank's balanced salt solution (HBSS) and weighed. These depots together comprise almost all visible adipose tissue and can be used to calculate fatness (fresh weight of adipose tissue as percentage live body mass). The popliteal lymph nodes, and four conspicuous mesenteric lymph nodes, were also dissected out and weighed. Samples of perinodal (within $2 \mathrm{~mm}$ the popliteal lymph nodes), 'middle' (about $5 \mathrm{~mm}$ from the nodes) and 'remote' (about $10 \mathrm{~mm}$ from the lymph nodes) from both popliteal depots and from the nodeless perirenal depots were dissected, using established procedures (Mattacks \& Pond, 1997, 1999; Mattacks et al. 2002, 2003). The average yield of perinodal adipose tissue from each popliteal depot was only $27 \mathrm{mg}$ wet weight, so all methods were adapted for use on such small samples. Two sets of corresponding intra-abdominal samples were defined by their anatomical relationships to lymphoid tissues; 'perinodal' and 'remote' adipose tissue from the mesentery and samples of the omentum that are rich in or lack milky spots. The former is equivalent to perinodal, and the latter to remote-from-node. Samples were also taken from the large perirenal depot, which in rats does not contain any lymph nodes.

The dissection was completed within $1 \mathrm{~h}$ of death and procedures to collect DC were begun immediately.

\section{Quantification of dendritic cells}

The $50 \mathrm{mg}$ adipose tissue samples were cut into $1-2 \mathrm{~mm}^{2}$ fragments and washed for $5 \mathrm{~min}$ in HBSS containing the chemokine C6kine $(1 \mathrm{ng} / \mathrm{ml})$ (CCL21; R \& $\mathrm{D}$ Systems, Abingdon, UK) that stimulates migration of DC 


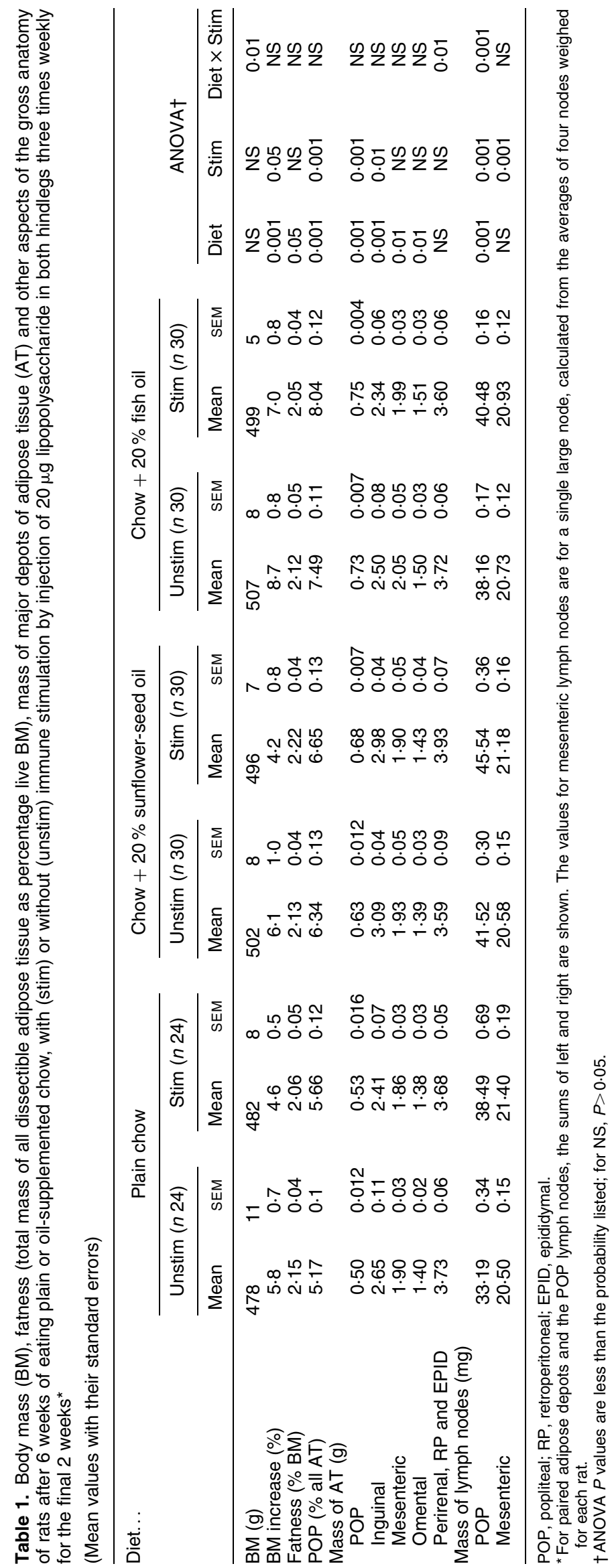




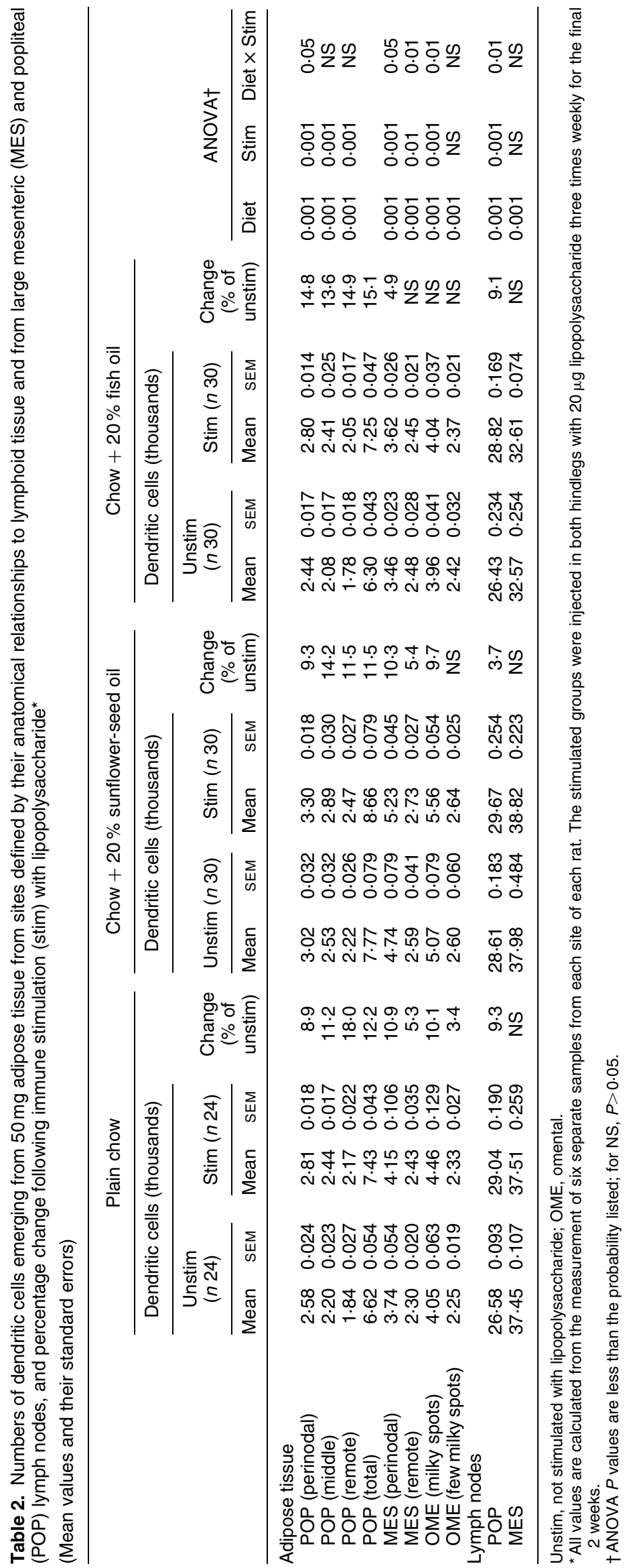


(Saeki et al. 1999; Luft et al. 2002), and 0.1\% bovine serum albumin. After rinsing with HBSS, each adipose sample and the lymph nodes, opened to release their contents, were resuspended in $5 \mathrm{ml}$ HBSS with $0.1 \%$ bovine serum albumin in $15 \mathrm{ml}$ Falcon tubes. The tubes were placed into a waterbath shaking at 100 cycles/min at $37^{\circ} \mathrm{C}$ for $4 \mathrm{~h}$, allowing the DC to fall to the base of the tube. After incubation, the adipose tissue and $4.5 \mathrm{ml}$ media were removed from each sample, leaving the DC in $0.5 \mathrm{ml}$. They were washed three times by filtering with a $10 \mu \mathrm{m}$ centrifuge filter and re-suspended in $0.5 \mathrm{ml} \mathrm{HBSS}+0.1 \%$ bovine serum albumin. DC were identified by their size, distinctive crinkled outline and by their selective binding of specific antibodies (Hill et al. 1990). The DC suspended in $0.5 \mathrm{ml} \mathrm{HBSS}+0.1 \%$ bovine serum albumin were identified by staining for $5 \mathrm{~min}$ with $0.01 \%$ peroxidase indicator reagent (Sigma) and a 1:20 solution of FITC anti-rat MHC-II (I-E) marker (BD Biosciences (Pharmingen), Oxford, UK). The cells were counted with a haemocytometer using bright field and fluorescence microscopy, to a precision of \pm 10 , from six separate $10 \mu$ l samples from each mesenteric and omental adipose tissue preparation and the lymph nodes, and three from each popliteal adipose site (pooling material from the left and right homologous sites). A sample of perirenal adipose tissue was also examined. The results were calculated as DC per $0.1 \mathrm{ml}$ (i.e. those that migrated from $50 \mathrm{mg}$ adipose tissue).

The left and right large popliteal lymph nodes and two large mesenteric lymph nodes were cut open, and the leucocytes released into HBSS. Each sample of leucocytes was centrifuged and layered on Histopaque (Sigma) and the DC were separated from the other classes of leucocytes, and counted using the method described above.

Data were analysed using the PC version of the Statistical Package for Social Sciences (SPSS Inc., Chicago, IL, USA).

\section{Results}

Gross anatomy

The mean values of the measures of body composition are shown in Table 1. These rats were healthy and free from visible wounds or infection, and the dose of LPS was less than $10 \%$ of that used to induce fever, so it is not surprising that all the rats gained a little weight during the experimental period, with the fish-oil-fed animals gaining slightly more than those eating sunflower-seed oil. ANOVA shows that the effects of LPS stimulation were slight and did not interact with diet. The experimental diets did not cause obesity in these mature adult rats; the final body masses and body composition (percentage fatness) of the unstimulated rats were not significantly different, though the LPS-injected rats eating sunflower-seed oil were slightly fatter (Student's $t$ test, $P<0.05$ ) than the corresponding group eating plain chow.

The detailed measurements of the masses of the adipose depots (Table 1) reveal that the body conformation was altered, albeit slightly, by the diet. The popliteal depots were larger, and comprised a greater proportion of the adipose tissue in all the oil-fed rats. In those fed fish oil, the other major lymphoid tissue-containing depots, the mesenteric and omental, were also slightly larger, but in those eating sunflower-seed oil, the main accumulation was in the inguinal depot that contains only a few localised lymph nodes. For each dietary group, the popliteal adipose depots were significantly larger in the LPS-stimulated specimens, by $8 \%$ in those fed chow supplemented with sunflower-seed oil, and by $3 \%$ in those eating fish oil. These increases are too large to be caused only by expansion of the embedded lymphoid tissue. Diet and LPS stimulation did not interact in determining the masses of any of these node-containing depots. The masses of the large nodeless perirenal and retroperitoneal, and epididymal depots are always variable in rats. The sums of the masses of these depots were not significantly altered by either type of treatment, with the minor interaction between factors probably spurious.

As expected, the popliteal lymph nodes that drained the area of skin where LPS was injected were significantly enlarged following the immune-stimulation regimen (Table 1). However, at a maximum of $16 \%$ (measured in the rats eating plain chow), the increase in node size was small, indicating that the injection regimen produced only mild, local inflammation. Nonetheless, ANOVA shows that diet interacted strongly with immune stimulation, with the popliteal nodes being $25 \%$ larger without LPS after the sunflower-seed-oil diet, and $15 \%$ larger after fish oil. In contrast, diet did not alter the mass of the mesenteric lymph nodes, and the increase was small (less than 5\%), and not significant for the fish-oil-fed rats (Student's $t$ test, $P>0.05$ compared with values from the rats on the plain diet). The absence of substantial differences in body size or fatness between the stimulated and unstimulated rats in any dietary group is also consistent with the conclusion that the injection regimen did not produce a major systemic immune response involving prolonged fever, anorexia or cachexia.

\section{Numbers of dendritic cells}

The migratory cells were identified as mostly mature and nearly mature DC by the procedures described above. The collection method used was designed to maximise the yield from small, site-specific samples of adipose tissue. C6kine preferentially stimulates DC but we cannot exclude the possibility that the samples included a few monocytes and macrophages or other types of similarsized leucocytes. The data in Table 2 show that only about $2-6 \times 10^{3}$ DC were collected from $50 \mathrm{mg}$ adipose tissue in depots associated with lymphoid tissue, not enough in each sample to permit further identification. No detectable DC were collected from the perirenal adipose tissue under any of the experimental conditions.

The proportions of DC in the three popliteal adipose samples were similar in the rats of all dietary groups, with $38-39 \%$ of those sampled found in the perinodal tissue, and 28-29\% in the remote-from-node sites (Table 2). The numbers of DC in the mesenteric perinodal and omental milky spot samples were consistently larger (by up to $69 \%$ ) than those collected from the popliteal perinodal adipose tissue of the same rats. Values from 
mesenteric and omental 'remote' sites of the rats eating plain chow were similar to those of popliteal 'middle', and higher than those of the popliteal 'remote' site. These site-specific properties indicate that the popliteal adipose depot contains fewer DC than either of the two lymphoid-tissue-containing intra-abdominal depots. In the rats fed plain chow, significant increases in DC were measured from all the adipose samples listed in Table 1, with the middle and remote popliteal samples that had the fewest DC in the quiescent state increasing the most.

The experimental diets altered numbers of DC in adipose tissue as much or more than stimulation with LPS (Table 2 ), with all values except those from mesenteric and omentum tissue remote from nodes or milky spots highly significantly different from the corresponding controls. All measurements except those for the mesenteric lymph nodes were $17-18 \%$ higher for samples from the unstimulated rats that ate sunflower-seed oil than for the corresponding samples from the rats on the other two diets. Following the sunflower-seed-oil diet, the total numbers of DC collected from the popliteal depot were greater without immune stimulation than with local stimulation in the rats eating the other diets (Student's $t$ test, $P<0.001$ ). Numbers of DC in all other adipose samples studied were also higher, by $25-29 \%$ for the perinodal samples of the intra-abdominal depots. These values are substantially greater than the increase elicited by LPS stimulation and indicate that more abundant DC in node-containing adipose tissue of the rats eating the sunflower-seed-oil diet could represent higher levels of immune activation. However, ANOVA shows that interactions between diet and immune stimulation were minor, in much of the popliteal not significant.

The fish-oil diet had an opposite, and smaller, effect on DC numbers in popliteal samples, depressing values by about $5 \%$ compared with the corresponding samples from the rats eating plain chow. However, immune stimulation increased DC numbers in the popliteal adipose tissue more following the fish-oil diet than in the samples from the control groups eating plain chow, making the average values about equal in corresponding samples from the two groups of rats. The most striking effect of the experimental diets was their actions on DC in the locations remote from the site of LPS stimulation, mesenteric and omental adipose tissue. Following the local stimulation of the popliteal depot, the 'remote' mesenteric and omental sites from the rats fed the sunflower-seed-oil diet contained only $12-13 \%$ more DC than the corresponding samples from the rats eating plain chow. The numbers of DC in both omental samples were unchanged by immune stimulation in the rats that had been eating fish oil, though they increased by about $10 \%$ in the milky spot-rich part of the omentum in the rats of the other dietary groups. Dietary lipids and mild immune stimulation altered total DC numbers but not their partitioning within the adipose depot containing the stimulated lymph nodes, suggesting that these factors modulate the formation and/or maturation of DC but not their rate of migration.

Table 2 also shows the numbers of DC collected from the major lymph nodes. Slightly $(7.6 \%)$ more DC emanated from the unstimulated popliteal nodes of the rats fed the sunflower-seed-oil diet. The maxima following immune stimulation were all similar, although $17 \%$ more DC were found in the contiguous perinodal adipose tissue. As in the case of DC in adipose tissue, numbers of DC emanating from the mesenteric nodes were strongly affected by diet. The fish-oil diet slightly (5\%) depressed numbers of DC recovered from mesenteric lymph nodes relative to those measured after the control diet, and from the adjacent perinodal adipose tissue, at least in the perinodal samples. However, although DC numbers in mesenteric perinodal adipose tissue were $27 \%$ higher in the rats eating sunflower-seed oil, no significant difference was recorded in numbers of DC from the nodes themselves. Following immune stimulation, more DC were collected from the popliteal lymph nodes, but, although the mesenteric lymph nodes were slightly enlarged following immune stimulation in two of the three groups of rats (see Table 1), the numbers of DC collected from them did not change significantly (Table 2).

Table 3 shows the correlations between the numbers of DC found in the adipose tissue samples and lymph nodes taken from Table 2, for the rats fed the two contrasting oil-enriched diets. As expected from Table 2, for both dietary groups, the values for the three popliteal samples correlate strongly with each other and less strongly but still

Table 3. Correlation coefficients $(r)$ between the numbers of dendritic cells extracted from adipose tissue samples and lymph nodes for all the rats fed sunflower-seed oil (upper right sector) and fish oil (lower left sector) $\dagger$

\begin{tabular}{|c|c|c|c|c|c|c|c|c|c|}
\hline & \multicolumn{4}{|c|}{ POP } & \multicolumn{3}{|c|}{ MES } & \multicolumn{2}{|c|}{ OME } \\
\hline & Perinodal & Middle & Remote & Lymph node & Perinodal & Remote & Lymph node & Milky spots & Few MS \\
\hline POP (perinodal) & & $0.831^{\star \star}$ & $0.792^{\star \star}$ & $0 \cdot 616^{\star \star}$ & $0.447^{\star \star}$ & $0.378^{\star *}$ & 0.207 & $0.610^{\star \star}$ & $0.490^{\star *}$ \\
\hline POP (middle) & $0.853^{\star \star}$ & & $0.810^{\star \star}$ & $0.504^{\star \star}$ & $0.441^{\star \star}$ & $0.474^{\star \star}$ & 0.145 & $0.511^{\star \star}$ & $0.388^{\star \star}$ \\
\hline POP (remote) & $0.859^{\star \star}$ & $0.852^{\star *}$ & & $0.576^{\star \star}$ & $0.543^{\star \star}$ & $0.421^{\star \star}$ & $0.275^{\star}$ & $0.640^{\star *}$ & $0.339^{\star \star}$ \\
\hline POP (lymph node) & $0.732^{* *}$ & $0.710^{* *}$ & $0.659^{\star \star}$ & & $0.468^{\star \star}$ & $0.337^{\star \star}$ & $0.342^{\star \star}$ & $0.514^{\star *}$ & $0.385^{\star \star}$ \\
\hline MES (perinodal) & $0.536^{\star \star}$ & $0.590^{\star *}$ & $0.572^{\star \star}$ & $0.507^{\star \star}$ & & 0.132 & $0.723^{\star \star}$ & $0.732^{\star \star}$ & 0.114 \\
\hline MES (remote) & -0.099 & -0.016 & -0.076 & 0.051 & 0.219 & & 0.081 & 0.198 & $0.495^{\star *}$ \\
\hline MES (lymph node) & 0.032 & -0.014 & 0.115 & $-0.282^{*}$ & 0.033 & -0.173 & & $0.548^{\star \star}$ & 0.150 \\
\hline OME (milky spots) & 0.118 & 0.173 & $0.291^{*}$ & 0.179 & 0.225 & $-0.269^{\star}$ & 0.177 & & $0.256^{\star}$ \\
\hline OME (few MS) & -0.147 & 0.036 & -0.033 & 0.004 & 0.148 & $0.510^{\star \star}$ & 0.181 & 0.104 & \\
\hline
\end{tabular}

POP, popliteal; MES, mesenteric; OME, omental; MS, milky spots.

${ }^{\star} P<0.05 ;{ }^{\star \star} P<0.01$.

†For the numbers of dendritic cells, see Table 2. There were thirty unstimulated and thirty lipopolysaccharide-injected rats in each group. 
highly significantly with the values from the adjacent popliteal lymph node and mesenteric perinodal. In the case of the rats fed fish oil, the only other strong correlation is that between the mesenteric and omental remote samples. The correlation between numbers of DC in the mesenteric and popliteal lymph nodes is actually negative. In contrast, following the sunflower-seed-oil diet, the corresponding correlation between numbers of DC in the two lymph nodes is positive and significant at $P<0.01$, and all the values from mesenteric and omental adipose tissue correlate with those of the popliteal adipose samples. Values from the mesenteric lymph nodes correlate strongly with those of the omental milky spots in the sunflowerseed-oil-fed rats only. The most 'independent' sample is the mesenteric remote, in which numbers of DC do not correlate with values from the adjacent perinodal sample, or that from the lymph nodes embedded in that depot.

\section{Discussion}

The advantage of starting with fully grown rats is that oilenriched diets fed ad libitum do not lead to greater fatness as they do in young rats; the mean final body masses and fatnesses of the groups of rats were only slightly different (Table 1). However, sex differences in the actions of steroid hormones and immune processes (Tanriverdi et al. 2003) mean that the conclusions from these data apply only to males.

The mesentery, omentum and popliteal depots are all rich in lymphoid tissue and consist of adipocytes that interact strongly with lymphoid cells and cytokines (Mattacks et al. 2002; Pond \& Mattacks, 2002; Pond, 2003b). Additional lymphocytes have been demonstrated in the omental adipose tissue associated with the stomach a few days after immunisation (Mueller et al. 2003). Selective enlargement in node-containing depots following fish-oil feeding has not previously been noted, though in young, growing rats fed large quantities of fish oil, the 'generalpurpose' epididymal and retroperitoneal depots do not hypertrophy as much as the mesenteric and inguinal depots (Belzung et al. 1993). Diets enriched with sunflower-seed oil preferentially enlarged the mainly 'general-purpose' inguinal depots and the popliteals, though to a lesser extent than following the fish-oil diet (Table 1). One cause may be selective uptake of different types of fatty acids. Site-specific differences in the rates of incorporation of fatty acids derived from dietary lipids into adipocyte triacylglycerols have been recorded in guinea-pigs (Colby \& Pond, 1993).

The immune-stimulation regimen induced further enlargement of the popliteal depots; the increase in mass was much greater than could be explained by numbers of DC (which are minute compared with adipocytes), and is probably due to the formation of additional adipocytes in the popliteal depots around the experimentally stimulated lymph nodes (Table 1). Adipocytes in these depots become smaller but more numerous following 6 weeks of chronic immune stimulation of the embedded lymph nodes (Mattacks et al. 2003). The greater enlargement of the popliteal adipose tissue around the stimulated lymph nodes in the rats that had eaten sunflower-seed oil than in those fed fish oil is consistent with the conclusion (Mattacks et al. 2003) that invasion by lymphoid cells promotes the local expansion of adipose tissue. This process, and the greater numbers of DC in all adipose sites recorded following the sunflower-seed-oil diet (Table 2), may also account for the selective enlargement of the inguinal depot in this group of rats (Table 1). The inguinal lymph nodes in this depot are concentrated near its dorsal border, with the bulk of it lacking lymphoid structures. The diet enriched in $n-6$ fatty acids would promote more DC permeating further through this depot, stimulating adipose tissue expansion with or without immune stimulation.

Dietary lipids modulated the numbers of DC in the mesenteric adipose tissue and the associated nodes much more than the numbers collected from the stimulated popliteal nodes. The responses of the milky spot region of the omentum to the experimental treatments resemble those of the mesenteric lymph nodes. The correlation between DC numbers from the popliteal and mesenteric lymph nodes is surprisingly weak, much weaker than that for the corresponding perinodal adipose tissue (Table 3). These findings are consistent with previous studies (Mattacks et al. 2002; Pond \& Mattacks, 2002) that suggest fundamental differences between the perinodal adipose tissue in the two anatomical sites. DC associated with mesenteric nodes seem to be continuously active (Huang et al. 2000), but there are no direct comparisons between those in popliteal and mesenteric lymph nodes. There may be intrinsic differences in lipid uptake or metabolism between the intra-abdominal nodes and the superficial popliteal. Mammary cells selectively take up $n-3$ fatty acids from the blood and secrete them as milk triacylglycerols (Lauritzen et al. 2001). A similar capacity in lymphoid cells would partially emancipate them from the effects of changes in dietary lipids.

The greatest effect on numbers of DC extracted from adipose tissue in the present experiment proved to be diet (Table 2), rather than immune stimulation. DC become functional about $3 \mathrm{~d}$ after the final division of their precursor cells and they continue to mature for several days (Randolph et al. 1998). The process is hastened by exposure to LPS (Nestle et al. 2001) and entails the secretion of eicosanoids derived from arachidonic acid (20:4n-6) (Harizi \& Gualde, 2002). The eicosanoid prostaglandin $\mathrm{E}_{2}$ is essential for C6kine-stimulated migration of DC in vitro (Scandella et al. 2002). The synthesis of such messenger molecules may be facilitated by diets enriched with oils such as sunflower-seed oil that contain a high proportion of $n-6$ PUFA. Dietary lipids have been shown to modulate their antigenpresenting capacities within 6 weeks (Sanderson et al. 1997). As we have found previously (Mattacks et al. 2002), dietary lipids modulate the spread of immune activation between distant adipose depots. Repeated immune stimulation also failed to raise lipolysis in the mesenteric and omental remote sites to the same extent as it did in the popliteal remote sites (Mattacks et al. 2002; Pond \& Mattacks, 2002). Following several weeks of the fish-oil diet, involvement of the mesentery and omentum in immune responses elicited in the popliteal depot is attenuated, whether measured by cytokine-stimulated lipolysis (Mattacks et al. 2002), or numbers of DC (Table 2). 
The pattern of site-specific differences in the action of dietary lipids on numbers of DC closely resembles that of sitespecific differences in lipolysis from adipocytes associated with lymph nodes during an acute immune response (Mattacks et al. 2002). This similarity is consistent with the conclusion that DC may mediate cytokine-induced lipolysis in adipocytes. DC may also take up some of the products of lipolysis for use as fuel and/or incorporation.

Table 3 provides further evidence that mesenteric perinodal, omental milky spots and the whole of the popliteal adipose depot share common properties. In contrast, the correlations between values from the mesenteric perinodal and omental milky spots and the corresponding remote sites in the same depots were much weaker, although these sites are anatomically nearer to each other. The differences between the two dietary groups in the involvement of the other adipose samples studied (except the nodeless perirenal depot) were striking.

These actions of dietary lipids on DC in tissues remote from the primary site of inflammation may be a major mechanism of the anti-inflammatory effects of fish oils, which have a high $n-3: n-6$ fatty acids ratio, and the contrasting action of oils such as sunflower-seed oil with a low $n-3: n-6$ fatty acids ratio. The action of PUFA on toll-like receptors (Lee et al. 2003) is unlikely to be the sole mechanism because the dietary lipids modulated numbers of DC even in the absence of LPS stimulation (Table 2). DC also have adrenergic receptors and their movements can be modulated by noradrenaline (Maestroni, 2000). Dietary lipids also alter noradrenaline-controlled lipolysis from popliteal adipocytes and others closely associated with lymphoid tissues, though only to a minimal extent in adipocytes from 'general-purpose' depots (Mattacks et al. 2002).

Perinodal adipocytes are the major source of fatty acids for lymph node lymphoid cells (Pond \& Mattacks, 2003). They incorporate a greater proportion of PUFA, but their composition is altered by diet, albeit more slowly than that of the 'general-purpose' adipocytes not associated with lymphoid tissue (Mattacks \& Pond, 1997). The fatty acid composition of the outer cell membrane, particularly the abundance and composition of their PUFA, determines the manifestation of immunologically important proteins (Jenski, 2000), and the recognition by T-cells of glycolipids associated with antigen-presenting molecules, as well as other proteins (De Libero \& Mori, 2003). The increased contact between DC and adipocytes associated with lymph nodes following the sunflower-seed-oilenriched diet (Table 2) suggests that switching from diets rich in $n-3$ fatty acids to diets rich in $n-6$ fatty acids may modulate immune function more quickly than the reverse change in diet.

DC are major mediators in HIV infection (Lo, 2003). Studies of animal models of AIDS suggest that altering the migration and other properties of DC is one of the ways that retroviruses, including HIV, evade destruction by the host's immune system, and foster opportunistic infections (Choi et al. 2003). In murine models of AIDS (Xi et al. 1998), and in human patients (Roman et al. 2001), the progress of viral infection can be modulated by dietary lipids, with fats such as fish oil, rich in $n-3$ fatty acids, curtailing the establishment and proliferation of the virus. Possible mechanisms include alterations of membrane structure and composition (Raulin, 2002). Lipid-rich diets characterised by a very low $n-3: n-6$ fatty acids ratio are common in developed countries, and are implicated in various abnormalities of immune function (Calder, 2001), including the increasing incidence of Crohn's disease, which is also characterised by an anomalous growth of mesenteric adipose tissue (Pond, 2001). Such diets increase the abundance of DC in intra-abdominal and peripheral adipose depots that incorporate lymphoid tissue (Table 2). They also preferentially modulate the mesenteric lymph nodes, which, together with other alimentary-associated lymphoid tissues, are major sites of proliferation of simian immunodeficiency virus and probably also HIV (O’Neil et al. 1999; Lopez et al. 2002). The greater numbers of DC in perinodal adipose tissue may facilitate the establishment of HIV in lymph nodes, and may promote the transfer of HIV to non-immune cells such as adipocytes where it may persist for long periods (Hazan et al. 2002). Hence diets rich in $n-6$ fatty acids may predispose individuals to infection with HIV and to some of its complications.

Most of the adipose depots that enlarge in HIV-associated adipose redistribution syndrome incorporate much lymphoid tissue, while those that shrink lack organised lymphoid organs (Pond, 2003b). The data in Table 2 show that they harbour more DC than nodeless depots. Infection with HIV alters the expression of cytokines in DC (Reinhart, 2003) which may modify their interactions with adipocytes as well as with other kinds of lymphoid cells in lymph nodes. The abnormal interactions between adipocytes and lymphoid cells arising from HIV infection and/or anti-retroviral drugs stimulate proliferation of adipocytes in depots that are anatomically contiguous to, and biochemically equipped to interact with, lymphoid cells (Mattacks et al. 2003). Many anti-retroviral drugs are toxic to adipocytes by various mechanisms, leading to lipo-atrophy (John et al. 2001). However, site-specific differences in the rates of these opposing processes would, slowly, lead to selective enlargement if inflammation-stimulated adipocyte proliferation is more vigorous, as in the mesenteric depot, or atrophy if poisoning by drugs predominates, as in superficial depots lacking embedded lymphoid tissue. Based on this hypothesis, diets rich in fish oil that curtail inflammation should attenuate the enlargement of depots that enclose lymphoid tissues infected with active HIV. Conversely, the condition may be exacerbated by diets rich in oils with a low $n-3: n-6$ fatty acids ratio such as sunflower-seed oil.

We conclude that dietary lipids modulate the abundance of DC in lymphoid tissue-containing adipose tissue both adjacent to and remote from stimulated lymph nodes. Fish oil limits detectable responses in tissues adjacent to the site of immune stimulation and in perinodal adipose tissue around other lymph nodes. Diets enriched with sunflower-seed oil with a low $n-3: n-6$ fatty acids ratio support more DC in adipose depots associated with lymphoid tissues, and the involvement of tissues anatomically distant from the site of immune stimulation in mild inflammatory responses. DC may mediate the exchange of signal 
molecules and nutrients between lymphoid and adipose cells. Perinodal adipose tissue and the DC therein function as integral components of the milky spots and lymph nodes.

\section{Acknowledgements}

This research was supported by a research grant to C. M. P. from Bristol-Myers Squibb (USA). We thank Dr Willem vas Dias, Seven Seas Ltd, Marfleet, Kingston upon Hull, UK for the gift of fish oil, and Ms Alison Sadler for making the oil-enriched diets. Professor Stella C. Knight and the Antigen Presentation Research Group, Northwick Park, Harrow, London provided invaluable advice. Dr R. H. Colby and an anonymous referee contributed helpful comments on the manuscript.

\section{References}

Bagga D, Wang L, Farias-Eisner R, Glaspy JA \& Reddy ST (2003) Differential effects of prostaglandin derived from $\omega-6$ and $\omega-3$ polyunsaturated fatty acids on $\mathrm{COX}-2$ expression and IL-6 secretion. Proc Natl Acad Sci U S A 100, 1751-1756.

Belzung F, Raclot T \& Groscolas R (1993) Fish oil $n$-3 fatty acids selectively limit hypertrophy of abdominal fat depots in growing rats fed high-fat diets. Am J Physiol 264, R1111-R1118.

Calder PC (2001) Polyunsaturated fatty acids, inflammation and immunity. Lipids 36, 1004-1024.

Choi YK, Fallert BA, Murphey-Corb MA \& Reinhart TA (2003) Simian immunodeficiency virus dramatically alters expression of homeostatic chemokines and dendritic cell markers during infection in vivo. Blood 101, 1684-1691.

Colby RH \& Pond CM (1993) Site-specific differences in the responses of guinea-pig adipose tissue to changes in the fatty acid composition of the diet. Nutr Res 13, 203-1212.

Cui L, Johkura K, Liang Y, Teng R, Ogiwara N, Okouchi Y, Asanuma K \& Sasaki K (2002) Biodefense function of omental milky spots through cell adhesion molecules and leukocyte proliferation. Cell Tissue Res 310, 321-330.

De Libero G \& Mori L (2003) Self glycosphingolipids: new antigens recognized by autoreactive T lymphocytes. News Physiol Sci 18, 71-76.

Harizi H \& Gualde N (2002) Dendritic cells produce eicosanoids, which modulate generation and functions of antigen-presenting cells. Prostagland Leukot Essent Fatty Acids 66, 459-466.

Hart DNJ (1997) Dendritic cells: unique leukocyte populations which control the primary immune response. Blood 90, 3245-3287.

Hazan U, Romero IA, Cancello R, et al. (2002) Human adipose cells express CD4, CXCR4, and CCR5 receptors: a new target cell type for the immunodeficiency virus-1? FASEB $J$ 16, U58-U71.

Hertz CJ, Kiertscher SM, Godowski PJ, Bouis DA, Norgard MV, Roth MD \& Modlin RL (2001) Microbial lipopeptides stimulate dendritic cell maturation via toll-like receptor 2. J Immunol 166, 2444-2450.

Hill S, Edwards AJ, Kimber I \& Knight SC (1990) Systemic migration of dendritic cells during contact sensitization. Immunology 71, 277-281.

Huang F-P, Platt N, Wykes M, Major JR, Powell TJ, Jenkins CD \& MacPherson GG (2000) A discrete subpopulation of dendritic cells transports apoptotic intestinal epithelial cells to T cell areas of mesenteric lymph nodes. J Exp Med 191, 435-443.

Jenski LJ (2000) Omega-3 fatty acids and the expression of membrane proteins: emphasis on molecules of immunologic importance. Curr Org Chem 4, 1185-1200.

John M, Nolan D \& Mallal S (2001) Antiretroviral therapy and the lipodystrophy syndrome. Antivir Therap 6, 9-20.

Lauritzen L, Hansen HS, Jørgensen MH \& Michaelsen KF (2001) The essentiality of long chain n-3 fatty acids in relation to development and function of the brain and retina. Prog Lipid Res 40, 1-94.

Lee JY, Plakidas A, Lee WH, Heikkinen A, Chanmugam P, Bray G \& Hwang DH (2003) Differential modulation of Toll-like receptors by fatty acids: preferential inhibition by $n$ 3 polyunsaturated fatty acids. J Lipid Res 44, 479-486.

Levine TP \& Chain BM (1992) Endocytosis by antigen presenting cells: dendritic cells are as endocytically active as other antigen presenting cells. Proc Natl Acad Sci U S A 89, 8342-8346.

Lo P (2003) HIV hijacks dendritic cells. Nat Med 9, 650.

Lopez MC, Huang DS \& Watson RR (2002) Changes in mesenteric lymph node $\mathrm{T}$ cell phenotype and $\mathrm{B}$ and $\mathrm{T}$ cell homing properties after murine AIDS infection. Lymphology 35, $76-86$.

Luft T, Jefford M, Luetjens P, Toy T, Hochrein H, Masterman KA, Maliszewski C, Shortman K, Cebon J \& Maraskovsky E (2002) Functionally distinct dendritic cell (DC) populations induced by physiologic stimuli: prostaglandin $\mathrm{E}_{2}$ regulates the migratory capacity of specific DC subsets. Blood 100, $1362-1372$.

Maestroni GJM (2000) Dendritic cell migration controlled by $\alpha_{1 \mathrm{~b}}$-adrenergic receptors. J Immunol 165, 6743-6747.

Mattacks CA \& Pond CM (1997) The effects of feeding suetenriched chow on site-specific differences in the composition of triacylglycerol fatty acids in adipose tissue and its interactions in vitro with lymphoid cells. Br J Nutr 77, 621-643.

Mattacks CA \& Pond CM (1999) Interactions of noradrenalin and tumour necrosis factor- $\alpha$, interleukin- 4 and interleukin- 6 in the control of lipolysis from adipocytes around lymph nodes. Cytokine 11, 334-346.

Mattacks CA, Sadler D \& Pond CM (2002) The effects of dietary lipids on adrenergically stimulated lipolysis in perinodal adipose tissue following prolonged activation of a single lymph node. Br J Nutr 87, 375-382

Mattacks CA, Sadler D \& Pond CM (2003) The cellular structure and lipid/protein composition of adipose tissue surrounding chronically stimulated lymph nodes in rats. J Anat Lond 202, $551-561$.

Morelli AE \& Thomson AW (2003) Dendritic cells under the spell of prostaglandins. Trends Immunol 24, 108-111.

Mueller A, O'Rourke J, Chu P, Kim CC, Sutton P, Lee A \& Falkow S (2003) Protective immunity against Helicobacter is characterized by a unique transcriptional signature. Proc Natl Acad Sci U S A 100, 12289-12294.

Nestle FO, Banchereau J \& Hart D (2001) Dendritic cells: on the move from bench to bedside. Nat Med 7, 761-765.

O’Neil SP, Mossman SP, Maul DH \& Hoover EA (1999) In vivo cell and tissue tropism of SIVsmmPBj14-bcl.3. AIDS Res Hum Retrovir 15, 203-215.

Pond CM (2001) Long-term changes in adipose tissue in human disease. Proc Nutr Soc 60, 365-374.

Pond CM (2003a) Paracrine interactions of mammalian adipose tissue. J Exp Zool 295A 99-110.

Pond CM (2003b) Paracrine relationships between adipose and lymphoid tissues: implications for the mechanism of HIVassociated adipose redistribution syndrome. Trends Immunol 24, 13-18.

Pond CM \& Mattacks CA (1995) Interactions between adipose tissue around lymph nodes and lymphoid cells in vitro. $J$ Lipid Res 36, 2219-2231.

Pond CM \& Mattacks CA (1998) In vivo evidence for the 
involvement of the adipose tissue surrounding lymph nodes in immune responses. Immunol Lett 63, 159-167.

Pond CM \& Mattacks CA (2002) The activation of adipose tissue associated with lymph nodes during the early stages of an immune response. Cytokine 17, 131-139.

Pond CM \& Mattacks CA (2003) The source of fatty acids incorporated into proliferating lymphoid cells in immune-stimulated lymph nodes. Br J Nutr 89, 375-382.

Randolph GJ, Beaulieu S, Lebecque S, Steinman RM \& Muller WA (1998) Differentiation of monocytes into dendritic cells in a model of transendothelial trafficking. Science 282, $480-483$.

Raulin J (2002) Human immunodeficiency virus and host cell lipids. Interesting pathways in research for a new HIV therapy. Prog Lipid Res 41, 27-65.

Reinhart TA (2003) Chemokine induction by HIV-1: recruitment to the cause. Trends Immunol 24, 351-353.

Roman DAD, Bachiller P, Izaola O, Romero E, Martin J, Arranz M, Bouza JME \& Aller R (2001) Nutritional treatment for acquired immunodeficiency virus infection using an enterotropic peptide-based formula enriched with $n-3$ fatty acids: a randomized prospective trial. Eur J Clin Nutr 55, $1048-1052$.

Saeki H, Moore AM, Brown MJ \& Hwang ST (1999) Cutting edge: secondary lymphoid-tissue chemokine (SLC) and CC chemokine receptor 7 (CCR7) participate in the emigration pathway of mature dendritic cells from the skin to regional lymph nodes. J Immunol 162, 2472-2475.
Sanderson P, MacPherson GG, Jenkins CH \& Calder PC (1997) Dietary fish oil diminishes the antigen presentation activity of rat dendritic cells. J Leukoc Biol 62, 771-777.

Scandella E, Men Y, Gillessen S, Forster R \& Groettrup M (2002) Prostaglandin E2 is a key factor for CCR7 surface expression and migration of monocyte-derived dendritic cells. Blood 100, 1354-1361.

Schreiner GF, Flye W, Brunt E, Korber K \& Lefkowith JB (1988) Essential fatty-acid depletion of renal-allografts and prevention of rejection. Science 240, 1032-1033.

Serhan CN, Hong S, Gronert K, Colgan SP, Devchand PR, Mirick G \& Moussignac RL (2002) Resolvins: a family of bioactive products of $\omega-3$ fatty acid transformation circuits initiated by aspirin treatment that counter proinflammation signals. J Exp Med 196, 1025-1037.

Smith JB \& Morris B (1970) The response of the popliteal lymph node of the sheep to swine influenza virus. Aust J Exp Biol Med 48, 47-55.

Tanriverdi F, Silveira LFG, MacColl GS \& Bouloux PMG (2003) The hypothalamic-pituitary-gonadal axis: immune function and autoimmunity. J Endocrinol 176, 293-304.

van Vugt E, van Rijthoven EAM, Kamperdijk EWA \& Beelen RHJ (1996) Omental milky spots in the local immune response in the peritoneal cavity of rats. Anat Rec 244, $235-245$.

Xi S, Cohen D \& Chen LH (1998) Effects of fish oil on cytokines and immune functions of mice with murine AIDS. J Lipid Res 39, $1677-1687$. 